\title{
Theory of valence-band holes as Luttinger spinors in vertically coupled quantum dots
}

\author{
J. I. Climente, ${ }^{1}$ M. Korkusinski, ${ }^{2}$ G. Goldoni, ${ }^{1,3}$ and P. Hawrylak ${ }^{2, *}$ \\ ${ }^{1}$ CNR-INFM National Center on nanoStructures and bioSystems at Surfaces (S3), Via Campi 213/A, 41100 Modena, Italy \\ ${ }^{2}$ Institute for Microstructural Sciences, National Research Council of Canada, Ottawa, Canada K1A OR6 \\ ${ }^{3}$ Dipartimento di Fisica, Università di Modena e Reggio Emilia, Via Campi 213/A, 41100 Modena, Italy \\ (Received 19 May 2008; revised manuscript received 11 August 2008; published 25 September 2008)
}

\begin{abstract}
We describe the valence-band holes of quantum dot molecules formed by two vertically coupled disks, using a four-band $k \cdot p$ Hamiltonian. It is shown that the strong spin-orbit coupling of the valence band introduces characteristic features in the hole tunneling, which are not captured by the usual single-band heavy-hole approximation. Therefore, a treatment of hole states as multiband Luttinger spinors is required. Within this description the parity symmetry in the vertical direction is lost, and chirality symmetry must be used instead. Effects of spin-orbit coupling on the hole and exciton states, as well as on the optical transitions are discussed. We show that, with increasing interdot distance, the spin-orbit interaction leads to a bonding-antibonding ground-state transition and to quenching of the excitonic emission. These results are relevant to recent experiments.
\end{abstract}

DOI: 10.1103/PhysRevB.78.115323

PACS number(s): 73.21.La, 73.40.Gk, 78.67.Hc

\section{INTRODUCTION}

There is current interest in engineering the electronic properties of quantum systems by coupling semiconductor quantum dots (QDs) and forming quantum molecules. In particular, molecules consisting of two vertically coupled quantum dots (CQDs) are being fabricated and investigated by several groups, ${ }^{1-10}$ often motivated by their possible application in quantum information storing through optical control of excitonic states. ${ }^{5,11-13}$ To date, the theoretical understanding of the electronic states in these structures is mostly based on single-band effective-mass approximations. ${ }^{1,3-11,14-19}$ While this model yields transparent results and is generally valid for conduction-band electrons, its validity for valenceband holes may be restricted. The reason for this is that the valence band is built from atomic $p$-type orbitals, and thus it is subject to a strong spin-orbit (SO) interaction, which leads to a sizeable coupling of heavy-hole $(\mathrm{HH})$ and light-hole (LH) subbands. ${ }^{20}$ In QDs this coupling is weakened by quantum confinement, which splits the HH and LH subbands. Thus, the lowest hole states of single QDs are in many aspects well described as single-band HH states. Understanding if this is also the case for CQDs is of interest, especially in the view of recent works pointing out the important role of hole tunneling in the formation of entangled exciton states. ${ }^{5-7,17,21}$

In a symmetric double dot molecule, tunneling allows the states, localized in the upper $(|\mathrm{QD} 1\rangle)$ and lower $(|\mathrm{QD} 2\rangle)$ dots, to hybridize, forming symmetric $(|s\rangle=|\mathrm{QD} 1\rangle+|\mathrm{QD} 2\rangle)$ and antisymmetric $(\mid$ as $\rangle=|\mathrm{QD} 1\rangle-|\mathrm{QD} 2\rangle)$ wave functions. From a simple Hubbard-like picture, the energy of these states is $E_{\mathrm{s}}=E_{0}-t$ and $E_{\mathrm{as}}=E_{0}+t$, where $E_{0}$ is the energy of the localized states and $t$ is the tunneling matrix element. ${ }^{3}$ For electrons, $t$ is always positive so that the ground state is symmetric and the first excited state is antisymmetric. This picture is essentially the same as that found in homonuclear diatomic molecules such as $H_{2}^{+}$. By contrast, for holes recent experiments have reported both positive and negative values of $t$. The latter implies the formation of exotic molecular ground states with strong antisymmetric character, which have no analogue in natural molecules and therefore open up perspectives of developing molecular systems with novel properties. ${ }^{22}$

Negative values of $t$ were predicted by a number of atomistic calculations, ${ }^{23-26}$ and its origin was related to the strong $p$-orbital component of the hole states. However, deeper understanding was hindered by the numerical nature of the studies. Moreover, these predictions referred to CQDs with pronounced structural and strain field asymmetries, which localize low-energy holes in one of the dots. In these heteronuclear-molecule-like systems, the atomic localization dominates over the molecular bond. Thus, the tunnel matrix elements are small and sensitive to fine atomistic effects. This is in contrast to the experimental system in Ref. 22, where resonant electric fields were used to tune the hole energy levels of the CQDs, forming strongly hybridized molecular states. These molecular states have a clear homonuclear-molecule-like character so that they may display large and robust tunneling elements. ${ }^{5,6}$ In spite of this, it was shown that with increasing interdot distance the value of $t$ switched from positive to negative. It was then suggested that this behavior could be ascribed to the SO interaction of holes, which modulates the tunneling as $t=t_{0}-t_{\mathrm{so}}$, where $t_{0}$ is the heavy-hole tunneling element and $t_{\mathrm{so}}$ the SO-induced correction.

In this paper, we use the $k \cdot p$ theory to provide detailed theoretical understanding of this and other effects of SO interaction on the hole states of CQDs. A four-band LuttingerKohn Hamiltonian is employed, which provides the simplest description while properly accounting for SO-induced subband mixing. ${ }^{27}$ Within this framework, the hole states are represented as Luttinger spinors, four-component objects with two HH and two LH states. The symmetries of the Luttinger spinors (and hence their derived physical properties) differ significantly from those of the single-band $\mathrm{HH}$ states. SO interaction couples spin and orbital degrees of freedom so the hole states cannot be classified by their spin but rather by a total angular momentum. Likewise, SO interaction breaks the conservation of the parity (inversion sym- 


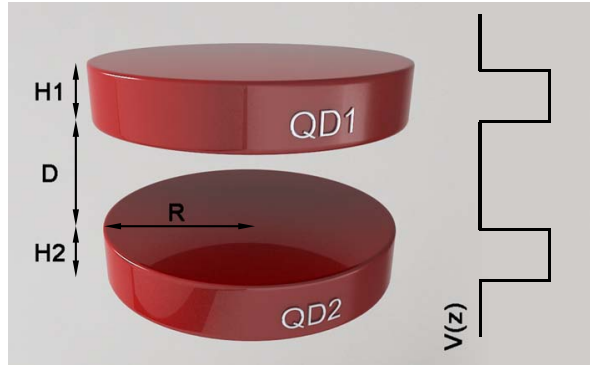

FIG. 1. (Color online) Schematic representation of the CQDs under study and the vertical confinement potential $\hat{V}^{h}(z)$.

metry in the vertical direction) so that the spinors contain a mixture of both symmetric and antisymmetric components even when the CQDs are identical. As an alternative to parity, we can classify the spinors by their chirality, which is isomorphic to the electronic spin quantum number. ${ }^{28,29}$

We calculate the hole states as a function of the interdot distance and show that SO interaction alone can explain the negative hole tunneling elements $t$ reported in Ref. 22. The SO interaction competes with the tunneling matrix element of the $\mathrm{HH}$, leading to an effective tunneling element, which may significantly differ from the single-band expectation. The conditions favoring negative tunneling elements are then elucidated. Some consequences of the resulting hole ground states (with strong antisymmetric character) are discussed, such as the formation of optically dark exciton ground states.

The paper is organized as follows: In Sec. II we give details of the theoretical model. Hole states and their tunneling are described in Sec. III. Section III A is devoted to the fundamental case of symmetric CQDs. Possible deviations from the previous predictions in self-assembled CQDs, owing to strain fields or to structural asymmetry, are studied in Sec. III B. In Sec. IV, we study the exciton states and their optical response as a function of the interdot distance. Conclusions are presented in Sec. V.

\section{THEORY}

We investigate the double quantum dot structure illustrated in Fig. 1. The dots are assumed to have disk shape with radius $R$, vertically separated by an interdot barrier of thickness $D$. The upper and lower dots, hereafter referred to as QD1 and QD2, have heights $H 1$ and $H 2$, respectively. Since we are interested in the tunneling along the vertical direction, we define the confining potential as $\hat{V}^{h}(\rho, z)$ $=\hat{V}^{h}(\rho)+\hat{V}^{h}(z)$, where $\hat{V}^{h}(\rho)$ is simply an infinite barrier in the radial direction, whereas $\hat{V}^{h}(z)$ is a square well potential, as shown on the right side of Fig. 1, whose height is the band-offset between the dot and barrier materials, $V_{c}$.

The valence band of semiconductors is built from $p$-type atomic orbitals. Therefore, the microscopic (Bloch) orbital angular momentum is $l=1$. Spin-orbit interaction couples $l$ with the spin quantum number $s=1 / 2$ so that hole states have a total Bloch angular momentum $J=l+s$, where $J$ $=3 / 2$ for $\mathrm{HH}$ and $\mathrm{LH}$ subbands, and $J=1 / 2$ for the split-off band. ${ }^{20}$ At the center of the Brillouin zone, the split-off band is energetically separated from the $\mathrm{HH}$ and $\mathrm{LH}$ subbands. As a result, the four-band Luttinger-Kohn Hamiltonian provides a good description of low-lying hole states by considering the coupling between $\mathrm{HH}\left(J=3 / 2, J_{z}= \pm 3 / 2\right)$ and $\mathrm{LH}(J$ $=3 / 2, J_{z}= \pm 1 / 2$ ) subbands while excluding the split-off band. This validity of this approximation is endorsed by the qualitative agreement between the four-band Luttinger spinor results and atomistic tight-binding calculations including the effect of remote bands. ${ }^{22,30}$ When spanned in the basis $J_{z}$ $=+3 / 2,-1 / 2,+1 / 2,-3 / 2$, the four-band Hamiltonian $\operatorname{reads}^{27,29}$

$$
\hat{\mathcal{H}}_{L K}=\left(\begin{array}{cccc}
\hat{P}_{+} & \hat{R} & -\hat{S} & 0 \\
\hat{R}^{*} & \hat{P}_{-} & 0 & \hat{S} \\
-\hat{S}^{*} & 0 & \hat{P}_{-} & \hat{R} \\
0 & \hat{S}^{*} & \hat{R}^{*} & \hat{P}_{+}
\end{array}\right)+\hat{V}^{h}(\rho, z) \mathcal{I},
$$

where $\mathcal{I}$ is the identity matrix. The operators in the above expression are given by

$$
\begin{gathered}
\hat{P}_{+}=\frac{\hbar^{2}}{2}\left[\left(\gamma_{1}+\gamma_{2}\right) \hat{p}_{\perp}^{2}+\left(\gamma_{1}-2 \gamma_{2}\right) \hat{p}_{z}^{2}\right], \\
\hat{P}_{-}=\frac{\hbar^{2}}{2}\left[\left(\gamma_{1}-\gamma_{2}\right) \hat{p}_{\perp}^{2}+\left(\gamma_{1}+2 \gamma_{2}\right) \hat{p}_{z}^{2}\right], \\
\hat{R}=\frac{\hbar^{2}}{2}(-\sqrt{3}) \gamma_{2} \hat{p}_{-}^{2}, \\
\hat{S}=\frac{\hbar^{2}}{2}(2 \sqrt{3}) \gamma_{3} \hat{p}_{-} \hat{p}_{z},
\end{gathered}
$$

with the Luttinger parameters $\gamma_{1}, \gamma_{2}$, and $\gamma_{3}$, and the operators $\hat{p}_{z}=-i \nabla_{z}, \hat{p}_{ \pm}=-i\left(\nabla_{x} \pm i \nabla_{y}\right)$, and $\hat{p}_{\perp}^{2}=\hat{p}_{x}^{2}+\hat{p}_{y}^{2}$.

The resulting hole eigenstates, known as Luttinger spinors, are four-component objects, each component associated to a different Bloch function $\left|J_{z}\right\rangle$. Owing to the rotational symmetry of the CQD system, the envelope part of each component has a well-defined azimuthal quantum number $m_{z}$. However, SO coupling mixes components with different $m_{z}$, and only the total angular momentum $F_{z}=m_{z}+J_{z}$ is a good quantum number for the entire spinor. ${ }^{29}$ The $k$ th hole state with a given $F_{z}$ can then be written as

$$
\left|F_{z}, k\right\rangle=\sum_{n, l}\left(\begin{array}{c}
A_{+3 / 2, n, l}^{F_{z}, k} f_{F_{z}-3 / 2, n}(\rho, \theta) \xi_{l}(z)\left|J_{z}=+\frac{3}{2}\right\rangle \\
A_{-1 / 2, n, l}^{F_{z}, k} f_{F_{z}+1 / 2, n}(\rho, \theta) \xi_{l}(z)\left|J_{z}=-\frac{1}{2}\right\rangle \\
A_{+1 / 2, n, l}^{F_{z}, k} f_{F_{z}-1 / 2, n}(\rho, \theta) \xi_{l}(z)\left|J_{z}=+\frac{1}{2}\right\rangle \\
A_{-3 / 2, n, l}^{F_{z}, k} f_{F_{z}+3 / 2, n}(\rho, \theta) \xi_{l}(z)\left|J_{z}=-\frac{3}{2}\right\rangle
\end{array}\right),
$$

where $f_{m_{z}, n}(\rho, \theta)$ is the in-plane envelope part,

$$
f_{m_{z}, n}(\rho, \theta)=\frac{e^{i m_{z} \theta}}{\sqrt{2 \pi}} \frac{\sqrt{2}}{R} \frac{J_{m_{z}}\left(k_{n}^{m_{z}} \rho\right)}{\left|J_{m_{z}+1}\left(k_{n}^{m_{z}} R\right)\right|} .
$$

$J_{m_{z}}\left(k_{n}^{m_{z}} \rho\right)$ is the Bessel function of order $m_{z}$ and radial quantum number $n . k_{n}^{m_{z}}$ represents the hole wave vector, de- 
fined in terms of the Bessel function roots $\left(\alpha_{n}^{m_{z}}\right)$ as $k_{n}^{m_{z}}$ $=\alpha_{n}^{m_{z}} / R$.

The vertical components in Eq. (3) are trigonometric functions, $\xi_{l}(z)=\sqrt{\frac{2}{W}} \cos \left(\frac{l \pi z}{W}\right)$ for $l$ odd and $\xi_{l}(z)=\sqrt{\frac{2}{W}} \sin \left(\frac{l \pi z}{W}\right)$ for $l$ even, where $W$ represents the size of the computational box along $z$. A proper linear combination of these functions may fit any double dot wave function in the vertical direction. In the particular case where the quantum molecule displays $z$-reflection symmetry (i.e., $H 1=H 2$ ), the diagonal terms of Hamiltonian (1) commute with the vertical inversion symmetry operator $\hat{i}_{z}$. Therefore, the individual components of the spinor have well-defined parity symmetry $\nu_{z}$, either symmetric $\left(\nu_{z}=\mathrm{s}\right)$ or antisymmetric $\left(\nu_{z}=\mathrm{as}\right)$. For symmetric components, only $\xi_{l}$ functions with $l$ odd (i.e., cosines) have nonzero coefficients, while for antisymmetric components, only $\xi_{l}$ with $l$ even (i.e. sines) do so. However, the off-diagonal SO terms of the Hamiltonian do not commute with $\hat{i}_{z}$, as $\left[\hat{i}_{z}, \hat{S}\right]=2 \hat{i}_{z} \hat{S}$. Thus, parity is not a good quantum number for the entire Luttinger spinor.

As an alternative to parity, we define the chirality operator $\hat{\sigma} \operatorname{as}^{28,29}$

$$
\hat{\sigma}=\left(\begin{array}{cccc}
\hat{i}_{z} & 0 & 0 & 0 \\
0 & \hat{i}_{z} & 0 & 0 \\
0 & 0 & -\hat{i}_{z} & 0 \\
0 & 0 & 0 & -\hat{i}_{z}
\end{array}\right),
$$

which can be envisaged as an isospin quantum number. ${ }^{28}$ Luttinger spinors are eigenfunctions of this operator with eigenvalue $\nu$. It follows from Eq. (5) that the two first and two last components of a Luttinger spinor with a defined chirality have opposite parity. We label the spinors as chirality up $(\nu=\uparrow)$, when the two first components are symmetric $\left(\nu_{z}=\mathrm{s}\right)$ and the two last are antisymmetric $\left(\nu_{z}=\mathrm{as}\right)$, and those with reversed order of parities as chirality down $(\nu=\downarrow)$. Thus, for $F_{z}=3 / 2$ the lowest $\nu=\uparrow$ state reads

$$
\begin{aligned}
|\nu=\uparrow\rangle_{h} & =\left|F_{z}=3 / 2, \nu=\uparrow, k=1\right\rangle \\
& =\sum_{n}\left(\begin{array}{cc}
\sum_{l=2 p-1} & A_{+3,2, n, l}^{3 / 2,1} f_{0, n}(\rho, \theta) \xi_{l}(z)\left|J_{z}=+\frac{3}{2}\right\rangle \\
\sum_{l=2 p-1} & A_{-1 / 2, n, l}^{3 / 2, \uparrow, 1} f_{2, n}(\rho, \theta) \xi_{l}(z)\left|J_{z}=-\frac{1}{2}\right\rangle \\
\sum_{l=2 p} & A_{+1 / 2, n, l}^{3 / 2, \uparrow, 1} f_{1, n}(\rho, \theta) \xi_{l}(z)\left|J_{z}=+\frac{1}{2}\right\rangle \\
\sum_{l=2 p} & A_{-3 / 2, n, l}^{3 / 2,1,1} f_{3, n}(\rho, \theta) \xi_{l}(z)\left|J_{z}=-\frac{3}{2}\right\rangle
\end{array}\right),
\end{aligned}
$$

where $p=1,2,3 \ldots$ is an integer. The corresponding $|\nu=\downarrow\rangle_{h}$ state reads the same but the parity of each component is reversed, so the order of the summations over $l$ is inverted.

Even though our theory is not restricted to a single class of material, for illustration purposes we will consider InGaAs/GaAs CQDs. Disk-shaped vertically aligned coupled quantum dots made of InGaAs with accurate control on the interdot distance are now grown by means of the indium flush technique. ${ }^{31}$ Moreover, there is evidence that the radius of the coupled dots may be very similar. ${ }^{17}$ The Luttinger

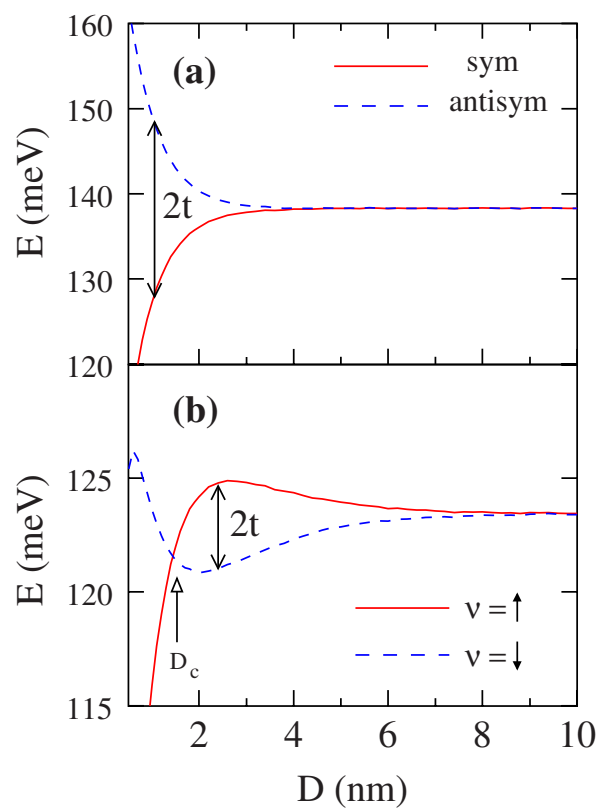

FIG. 2. (Color online) Energy levels of the two lowest hole states as a function of the interdot distance calculated as (a) singleband $\mathrm{HH}$ states and (b) Luttinger spinors. In (b), $D_{c}$ is the critical distance at which the tunneling element $t$ switches from positive to negative.

parameters for InGaAs are $\gamma_{1}=11.01, \gamma_{2}=4.18$, and $\gamma_{3}$ $=4.84$, and the valence-band offset $V_{c}=380 \mathrm{meV} .{ }^{32}$ The hole states of the CQD are calculated by exact diagonalization of $\hat{\mathcal{H}}_{\mathrm{LK}}$, on a basis with six radial states $(n=0-5)$ and 46 harmonics in the vertical direction $(l=1-46)$. The matrix elements of Hamiltonian (1) in the basis of Eq. (3) are given in Appendix B.

\section{HOLE STATES}

In this section we study the hole states in CQDs as a function of the structural parameters, focusing on the tunneling properties of the lowest-lying energy levels, i.e. the ground and first excited states with $F_{z}=3 / 2 .{ }^{33}$ These two states have the same radial part of the wave function but they differ in the vertical direction.

\section{A. Symmetric coupled quantum dots}

Figure 2 represents the energy spectrum vs interdot distance for a double dot with $R=10 \mathrm{~nm}$ and $H 1=H 2=2 \mathrm{~nm}$. Panel (a) shows the energy levels calculated with a singleband effective-mass Hamiltonian [i.e., setting the offdiagonal terms of Hamiltonian (1) to zero]. This is the usual approximation in most previous works. ${ }^{1,3-11,14-18}$ The ground and excited states correspond to the symmetric and antisymmetric HH states with $m_{z}=0$, respectively, which is consistent with a positive tunneling element $t$. As in the electron case, the energy splitting between the two states $(2 t)$ decreases exponentially with increasing interdot distance. . $^{3,19,34}$ A strikingly different behavior is however observed when the entire Hamiltonian is evaluated, as shown in panel (b). The 

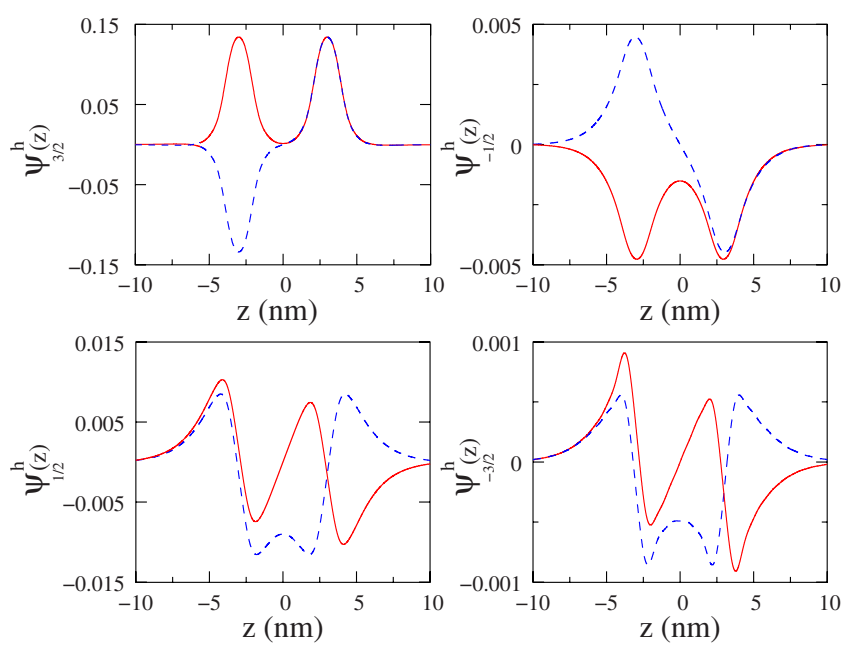

FIG. 3. (Color online) Wave-function profile along $z$ for the four components, $\Psi_{J_{z}}^{h}$, of the hole ground (dashed line) and first excited (solid line) states of Fig. 2(b) at $D=4 \mathrm{~nm}$. The ground-state chirality is $\nu=\downarrow$ and the excited state one is $\nu=\uparrow$. Note the opposite parity of the $J_{z}=+3 / 2,-1 / 2$ components as compared to the $J_{z}=+1 / 2$, $-3 / 2$ ones.

tunneling element $t$ no longer exhibits a monotonic trend as a function of $D$ but a rather intricate behavior. At small interdot distances, $t$ is positive and then the ground and first excited states have $\nu=\uparrow$ and $\nu=\downarrow$ symmetry, respectively. Yet, the tunneling element decreases quickly, and after a critical distance $D_{c}$ it becomes negative so that the ground state acquires $\nu=\downarrow$ symmetry. The negative tunneling element may be as large as a few millielectron volt, and only at larger interdot distances it decreases gradually.

The behavior observed in Fig. 2(b) agrees qualitatively with that inferred from the experiments and the theoretical simulations of Ref. 22. This is in spite of the fact that here we consider an ideal CQD system with no asymmetries induced by realistic confinement details or strain fields. It then follows that the reversal of the hole ground-state symmetry is a consequence of their Luttinger spinor nature, which can be understood without invoking atomistic effects or confinement potential-induced broken parity. In particular, the drastic change between Figs. 2(a) and 2(b) can be traced back to the off-diagonal SO operator $\hat{S}$ of the Luttinger-Kohn Hamiltonian, which mixes $\mathrm{HH}$ and LH states with opposite parity.

In Fig. 3 we depict the wave-function profile along the vertical direction for the four components of the Luttinger spinors of Fig. 2(b) at $D=4 \mathrm{~nm}$. Dashed lines are used for the $\nu=\downarrow$ ground state and solid lines for the $\nu=\uparrow$ excited state. One can see that the $J_{z}=+3 / 2$ and $J_{z}=-1 / 2$ components of the ground state are antisymmetric, while the $J_{z}$ $=+1 / 2$ and $J_{z}=-3 / 2$ are symmetric. The opposite set of parities holds for the excited state, owing to the different chirality. For both ground and excited states, the dominating component is by far the $J_{z}=+3 / 2 \mathrm{HH}$ (over ten times larger than the other components). This was expected because the quantum confinement in QDs shifts the LH subband towards high energies so that the low-energy levels are essentially weakly coupled $\mathrm{HH}^{20}$ It is then possible to define the $\nu=\downarrow$ spinor as "almost antisymmetric" and the $\nu=\uparrow$ one as "al-

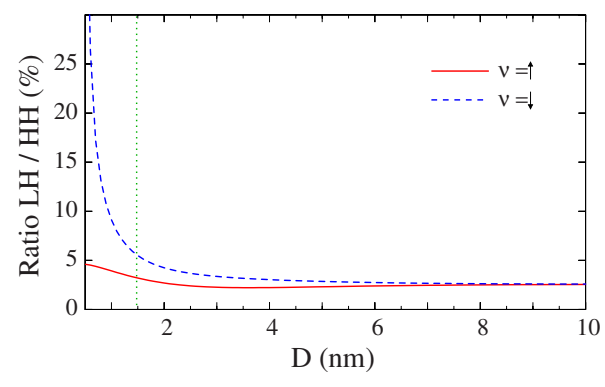

FIG. 4. (Color online) Ratio of LH vs HH weight in the chirality up and down spinors of Fig. 2(b). The vertical dashed line indicates the critical interdot distance at which the energy levels are reversed.

most symmetric." 35 Still, the minor components of the spinor cannot be neglected as they induce the important changes in the tunneling behavior observed in Fig. 2(b). It is worth noting here that the largest minor component is the LH with $J_{z}=+1 / 2$ because its envelope angular momentum $(m=1)$ is smaller than that of the other minor components [see Eq. (6)].

A more systematic view of the relative weight of the $\mathrm{LH}$ components is given in Fig. 4, where we plot the ratio of LH and $\mathrm{HH}$ weights in the Luttinger spinors of Fig. 2(b). The chirality up spinor (mostly a symmetric $\mathrm{HH}$ state) has a small $(<5 \%)$ contribution from the LHs, coming mainly from the $J_{z}=+1 / 2$ antisymmetric $\mathrm{LH}$ component. The chirality down spinor (mostly an antisymmetric $\mathrm{HH}$ ) has a larger LH contribution. This is because the $J_{z}=+1 / 2 \mathrm{com}-$ ponent is now a symmetric LH and, for strong tunneling, symmetric LH states are close in energy to the antisymmetric HHs. ${ }^{36}$ However, at the point of the ground-state reversal (vertical dashed line) both chirality up and down spinors have a small $(\$ 5 \%)$ LH weight, which remains small for longer distances. Thus, the chirality down ground state is not a consequence of an increasing $\mathrm{LH}$ weight.

To obtain a clearer insight into the effect of the SO interaction, we next build a simple semianalytical model, which allows us to disentangle the kinetic, potential, and SO interaction contributions to the hole energy. We redefine the potential along $z$ as two coupled parabolas centered in QD1 and QD2;

$$
\hat{V}^{h}(z)=\frac{1}{2} m_{\mathrm{HH}}^{*} \omega_{\mathrm{HH}}^{2} \min \left[\left(z-D_{1 / 2}\right)^{2},\left(z+D_{1 / 2}\right)^{2}\right],
$$

where $m_{\mathrm{HH}}^{*}$ is the $\mathrm{HH}$ effective mass, $m_{\mathrm{HH}}^{*}=\frac{1}{\gamma_{1}-2 \gamma_{2}}, \omega_{\mathrm{HH}}$ is the confining frequency of the parabolas, and $2 D_{1 / 2}$ is the distance between the minima of the parabolas. Note that both $\mathrm{HH}$ and LH components feel the same confinement potential, namely, Eq. (7). When the dots are far enough to be effectively isolated, the wave functions along $z$ are the eigenfunctions of the one-dimensional harmonic oscillator potentials,

$$
\xi_{j}^{\mathrm{QD} 1}(z)=\left(\frac{\alpha_{j}}{\pi}\right)^{1 / 4} e^{-\alpha_{j}\left(z-D_{1 / 2}\right)^{2} / 2}
$$




$$
\xi_{j}^{\mathrm{QD} 2}(z)=\left(\frac{\alpha_{j}}{\pi}\right)^{1 / 4} e^{-\alpha_{j}\left(z+D_{1 / 2}\right)^{2} / 2},
$$

where $\alpha_{j}=\frac{m_{j}^{*} \omega_{j}}{\hbar}$, with $j=\mathrm{HH}, \mathrm{LH}$. Here, $m_{\mathrm{LH}}^{*}=\frac{1}{\gamma_{1}+2 \gamma_{2}}$ and $\omega_{\mathrm{LH}}=\sqrt{\frac{m_{\mathrm{HH}}^{*}}{m_{\mathrm{LH}}^{*}}} \omega_{\mathrm{HH}}$. Since LHs are lighter than HHs, they penetrate more into the barrier $\left(\alpha_{\mathrm{LH}}>\alpha_{\mathrm{HH}}\right)$.

When the dots are closer together, tunneling couples $\xi_{j}^{\mathrm{QD} 1}(z)$ and $\xi_{j}^{\mathrm{QD} 2}(z)$, forming symmetric and antisymmetric combinations, $\xi_{j}^{\mathrm{s}}(z)=N_{j}^{\mathrm{s}}\left(\xi_{j}^{\mathrm{QD} 1}+\xi_{j}^{\mathrm{QD} 2}\right)$ and $\xi_{j}^{\mathrm{as}}(z)=N_{j}^{\mathrm{as}}\left(\xi_{j}^{\mathrm{QD} 1}\right.$ $\left.-\xi_{j}^{\mathrm{QD} 2}\right)$. Here, $N^{\mathrm{s}}$ and $N^{\mathrm{as}}$ stand for the normalization constants. An ansatz can then be postulated for the lowest $\nu=\uparrow$ $(\nu=\downarrow)$ wave function of the complete Luttinger-Kohn Hamiltonian, which preserves the chirality symmetry,

$$
\begin{aligned}
\mid F_{z} & \left.=\frac{3}{2}, \nu=\uparrow(\downarrow), k=1\right\rangle_{\text {parab }} \\
& =\sum_{n}\left\{\begin{array}{l}
A_{+3 / 2, n}^{3 / 2, \uparrow(\downarrow), 1} f_{0, n}(\rho, \theta) \xi_{\mathrm{HH}}^{\mathrm{s}}(z)\left[\xi_{\mathrm{HH}}^{\mathrm{as}}(z)\right]\left|J_{z}=+\frac{3}{2}\right\rangle \\
A_{-1 / 2, n}^{3 / 2, \uparrow(\downarrow), 1} f_{2, n}(\rho, \theta) \xi_{\mathrm{LH}}^{\mathrm{s}}(z)\left[\xi_{\mathrm{LH}}^{\mathrm{as}}(z)\right]\left|J_{z}=-\frac{1}{2}\right\rangle \\
A_{+1 / 2, n}^{3 / 2, \uparrow(\downarrow), 1} f_{1, n}(\rho, \theta) \xi_{\mathrm{LH}}^{\mathrm{as}}(z)\left[\xi_{\mathrm{LH}}^{\mathrm{s}}(z)\right]\left|J_{z}=+\frac{1}{2}\right\rangle \\
A_{-3 / 2, n}^{3 / 2, \uparrow(\downarrow), 1} f_{3, n}(\rho, \theta) \xi_{\mathrm{HH}}^{\mathrm{as}}(z)\left[\xi_{\mathrm{HH}}^{\mathrm{s}}(z)\right]\left|J_{z}=-\frac{3}{2}\right\rangle
\end{array}\right\},
\end{aligned}
$$

We calculate the expectation value of Hamiltonian (1), using Eq. (7) confinement potential, in the basis of Eq. (10). Since we are interested in understanding the tunneling behavior in the vertical direction, we only consider $n=0$, which yields the simplest expressions in the radial direction. Furthermore, we only consider the terms involving the dominant hole component, $J_{z}=+3 / 2$. Within this approximation, the energy of the $\nu=\uparrow$ state is

$$
\begin{aligned}
E^{\uparrow}= & \left(A_{3 / 2,0}^{3 / 2, \uparrow, 1}\right)^{2}\left\langle 0,0, \mathrm{~s}, \mathrm{HH}\left|\hat{P}_{+}+\hat{V}^{h}\right| 0,0, \mathrm{~s}, \mathrm{HH}\right\rangle \\
& +2 A_{3 / 2,0}^{3 / 2, \uparrow, 1} A_{-1 / 2,0}^{3 / 2, \uparrow}\langle 0,0, \mathrm{~s}, \mathrm{HH}|\hat{R}| 2,0, \mathrm{~s}, \mathrm{LH}\rangle \\
& -2 A_{3 / 2,0}^{3 / 2, \uparrow, 1} A_{1 / 2,0}^{3 / 2, \uparrow}\langle 0,0, \mathrm{~s}, \mathrm{HH}|\hat{S}| 1,0, \mathrm{as}, \mathrm{LH}\rangle,
\end{aligned}
$$

and that of the $\nu=\downarrow$ state,

$$
\begin{aligned}
E^{\downarrow}= & \left(A_{3 / 2,0}^{3 / 2, \downarrow, 1}\right)^{2}\left\langle 0,0, \text { as }, \mathrm{HH}\left|\hat{P}_{+}+\hat{V}^{h}\right| 0,0, \text { as }, \mathrm{HH}\right\rangle \\
& +2 A_{3 / 2,0}^{3 / 2, \downarrow, 1} A_{-1 / 2,0}^{3 / 2, \downarrow, 1}\langle 0,0, \text { as }, \mathrm{HH}|\hat{R}| 2,0, \text { as }, \mathrm{LH}\rangle \\
& -2 A_{3 / 2,0}^{3 / 2, \downarrow, 1} A_{1 / 2,0}^{\frac{3}{2}, \downarrow, 1}\langle 0,0, \text { as }, \mathrm{HH}|\hat{S}| 1,0, \mathrm{~s}, \mathrm{LH}\rangle .
\end{aligned}
$$

In the above expressions, $\left|m, n, \nu_{z}, j\right\rangle=f_{m, n}(\rho, \theta) \xi_{j}^{\nu_{z}}(z)$. The coefficients $A_{J_{z}, 0}$ are taken from exact numerical calculations and used as input for the analytical ansatz. The inplane part of the operator matrix elements is the same as in the numerical model, and the vertical part is evaluated by letting the operators $\hat{V}^{h}(z), \hat{p}_{z}^{2}$, and $\hat{p}_{z}$ act on $\xi^{\text {as }}(z)$ and $\xi^{\S}(z)$ (see Appendix B for further details).

The energy levels resulting from Eqs. (11) and (12) for a CQD with $R=10 \mathrm{~nm}$ and $H 1=H 2=5 \mathrm{~nm}$, as a function of the interdot distance, are shown in Fig. 5(a) using thin lines. Thick lines depict the exact numerical solution for comparison. Clearly, the semianalytical model is an upper bound in energy, but it suffices to reproduce the qualitative behavior of
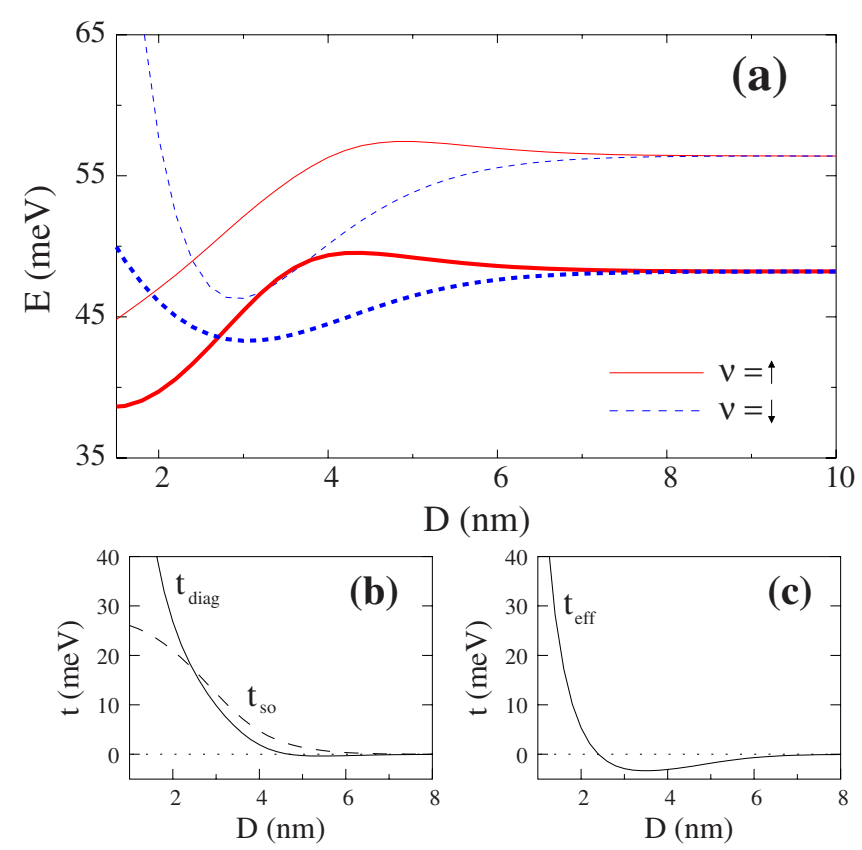

FIG. 5. (Color online) (a) Energy levels of the two lowest hole states as a function of the interdot distance calculated with the semianalytical (thin lines) and exact numerical (thick lines) model. (b) Diagonal tunneling element (solid line) and spin-orbit correction (dashed line). (c) Effective tunneling element adding the diagonal and spin-orbit tunneling terms. Panels (b) and (c) are derived from the semianalytical model. Dotted lines in (b) and (c) highlight the zero of energy.

the $\nu=\uparrow$ and $\nu=\downarrow$ states. Thus, we can use this model to investigate the influence of SO coupling. First, we define the diagonal tunneling element $t_{\mathrm{diag}}$,

$$
\begin{aligned}
t_{\mathrm{diag}}= & \frac{1}{2}\left[\left(A_{3 / 2,0}^{3 / 2, \downarrow, 1}\right)^{2}\left\langle 0,0, \mathrm{as}, \mathrm{HH}\left|\hat{P}_{+}+\hat{V}^{h}\right| 0,0, \text { as }, \mathrm{HH}\right\rangle\right. \\
& \left.-\left(A_{3 / 2,0}^{3 / 2, \uparrow, 1}\right)^{2}\left\langle 0,0, \mathrm{~s}, \mathrm{HH}\left|\hat{P}_{+}+\hat{V}^{h}\right| 0,0, \mathrm{~s}, \mathrm{HH}\right\rangle\right]
\end{aligned}
$$

which corresponds to half of the energy splitting between the $\nu=\downarrow$ (excited) state and the $\nu=\uparrow$ (ground) state neglecting SO terms. Actually, this is the tunneling element between symmetric and antisymmetric HH states [as in Fig. 2(a)]. Next, we shall compare it with the effective tunneling element, $t_{\text {eff }}$,

$$
t_{\text {eff }}=t_{\text {diag }}-t_{\text {so }}
$$

which properly includes the relevant SO term coupling components of the spinor with opposite parity, $t_{\mathrm{so}}$,

$$
\begin{aligned}
t_{\mathrm{so}}= & A_{3 / 2,0}^{3 / 2, \downarrow, 1} A_{1 / 2,0}^{3 / 2, \downarrow, 1}\langle 0,0, \text { as }, \mathrm{HH}|\hat{S}| 1,0, \mathrm{~s}, \mathrm{LH}\rangle \\
& -A_{3 / 2,0}^{3 / 2, \uparrow, 1} A_{1 / 2,0}^{3 / 2, \uparrow, 1}\langle 0,0, \mathrm{~s}, \mathrm{HH}|\hat{S}| 1,0, \text { as }, \mathrm{LH}\rangle .
\end{aligned}
$$

It is worth stressing that the SO term in Eq. (14) opposes the diagonal tunneling element (see Appendix A). Note also that $t_{\text {eff }}$ allows us to derive the energy levels of the double dot system simply as $E^{\uparrow}=E_{0}-t_{\text {eff }}$ and $E^{\downarrow}=E_{0}+t_{\text {eff }}$, where $E_{0}$ represents the energy of the hole localized in one dot. 


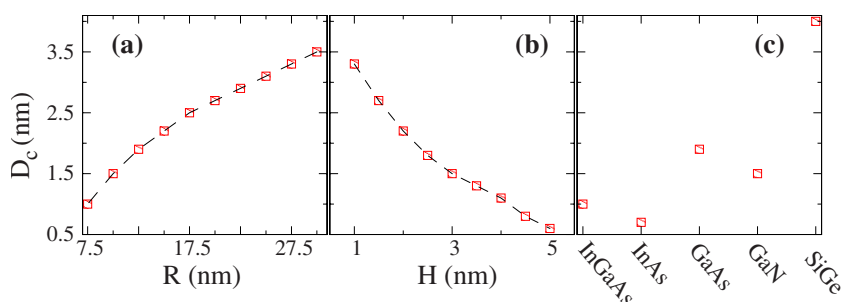

FIG. 6. (Color online) Interdot distance at which chirality $\nu=\uparrow$ and $\nu=\downarrow$ states cross as a function of the (a) disks radius, (b) height, and (c) material composition. In (a), the disks height is set to $H 1=H 2=2 \mathrm{~nm}$. In (b), the radius is set to $R=15 \mathrm{~nm}$. In (c), $R=10 \mathrm{~nm}$ and $H 1=H 2=3 \mathrm{~nm}$.

The diagonal tunneling element and the SO coupling term are compared in Fig. 5(b), using solid and dashed lines, respectively. As can be seen, $t_{\text {diag }}$ resembles the usual singleband element for it is positive and decreases exponentially until degeneracy is attained. On the other hand, $t_{\mathrm{so}}$ is just a correction for small interdot distances, when $t_{\text {diag }}$ is large. However, as the interdot distance increases it gains relative weight and eventually it becomes the dominant term. When this occurs, the effective tunneling element becomes negative [Fig. 5(c)] and the ground-state symmetry is reversed. The slower decay of $t_{\mathrm{so}}$ is partly due to its LH origin as compared to the HH origin of $t_{\text {diag }}$ [see Eqs. (13) and (15)] and partly due to the longer-range of the SO interaction as compared to the mechanical tunnel-coupling.

We are now in a position to explain the ground-state reversal in simple terms. The SO interaction introduces minor LH components to the otherwise pure $\mathrm{HH}$ states, forming Luttinger spinors [Eq. (3)]. Due to the chirality symmetry of the spinors, the dominant $\mathrm{HH}$ and the largest $\mathrm{LH}$ components have opposite parity [Eq. (6)]. Thus, the symmetric HH state is mixed with an antisymmetric LH state and the antisymmetric HH with a symmetric LH. Although the weight of these LH components remains small for most interdot distances (Fig. 4), their influence scales up as the two dots are separated because the tunneling of LHs decays much slower than that of HHs [Fig. 5(b)]. Thus, the symmetric HH is increasingly unstabilized by the antisymmetric $\mathrm{LH}$, while the antisymmetric HH is increasingly stabilized by the symmetric LH. At some point this leads to a reversal of the almost symmetric and almost antisymmetric states. A simple phenomenological model summarizing this is presented in Appendix A.

We next investigate the conditions favoring negative effective hole tunneling elements in CQDs, i.e., the conditions enhancing the relative weight of the SO terms, $\langle\hat{S}\rangle$. To elucidate the dependence on structural parameters of the disks, which constitute the CQD, in Fig. 6 we plot the critical interdot distance at which $\nu=\uparrow$ and $\nu=\downarrow$ levels cross $\left(D_{c}\right)$ as a function of the disks radius and height calculated with the numerical model. From the figure, it is apparent that the level reversal takes place at smaller interdot distances if the disks have small radius or large height. The radial dependence can be understood from the matrix element (B3) shown in the Appendix B, where one can see that $\hat{S}$ matrix elements are inversely proportional to $R$. As for the height dependence, it should be noted that with increasing disk height, the wavefunction penetration into the barrier diminishes and so does the tunneling energy. In addition, the diagonal kinetic energy proportional to $\hat{p}_{z}^{2}$ decreases faster than the off-diagonal spinorbit term proportional to $\hat{p}_{z}$. As a result, the relative contribution from the $\hat{S}$ operator increases. A straightforward material dependence can be also inferred from Eq. (2d). Negative $t_{\text {eff }}$ will be generally favored in systems with large spin-orbit interaction $\left(\gamma_{3}\right)$. This is confirmed in Fig. 6(c), where the critical distance is compared for different materials: InGaAs, InAs, GaAs, GaN (zinc blende), and SiGe. The Luttinger parameters are taken from Refs. 29 and 32; the disks have $R=10 \mathrm{~nm}$ and $H=3 \mathrm{~nm}$ and a band-offset of 200 $\mathrm{meV}$. While the level reversal is a rather general phenomenon, not restricted to the InGaAs system studied so far, the critical distance varies significantly depending on the material. Thus, almost antisymmetric ground states are found at short distances for InAs $\left(\gamma_{3}=9.2\right)$ and at longer distances as the Ga content increases; first InGaAs $\left(\gamma_{3}=4.84\right)$ and then GaAs $(\gamma=2.93)$. Indeed, we have checked that the level reversal is hardly observable in etched GaAs CQDs ${ }^{10}$ due to the large radius of the dots and the weak spin-orbit interaction.

\section{B. Strain and asymmetry in self-assembled coupled quantum dots}

From the above discussion, it is clear that usual $\operatorname{In}(\mathrm{Ga}) \mathrm{As} /$ GaAs CQDs are liable to display the characteristic multiband hole tunneling behavior, for spin-orbit mixing is strong in InAs systems and the dots have small radii. Here we evaluate two additional effects which are often present in selfassembled structures and were neglected in the previous discussion, namely, strain and broken symmetry of the CQD, to analyze possible departures from the ideal system.

Strain fields are fundamental in self-assembled dots grown by the Stranski-Krastanow method, ${ }^{20}$ and they play an essential role for the nucleation of dots on top of each other in order to form vertically coupled structures. ${ }^{37}$ Negative hole tunneling elements have been predicted for strained CQDs using atomistic models. ${ }^{22,24-26}$ Therefore, rather than carrying out a thorough analysis of the strain influence, we shall focus on its most relevant effects; the splitting between $\mathrm{HH}$ and LH subbands and the shallow confinement of LHs in the interdot barrier material. ${ }^{38}$

In InGaAs/GaAs QDs, biaxial strain may split $\mathrm{HH}$ and LH subbands by as much as $200 \mathrm{meV} \cdot{ }^{34}$ Obviously, this weakens the SO coupling between $\mathrm{HH}$ and $\mathrm{LH}$ subbands, up to the point where the ground state has almost exclusively $\mathrm{HH}$ character. This argument has been used by many authors to justify single-band $\mathrm{HH}$ approximations for self-assembled CQD structures. ${ }^{3-9,11,14-19}$ However, according to our previous results, it appears that even a small SO mixing suffices to induce drastic changes in the hole tunneling behavior. To test the validity of the single-band approximation in strained CQDs, in panels (a) and (b) of Fig. 7 we compare the energy of the lowest $\nu=\uparrow$ and $\nu=\downarrow$ states in a CQD with $R$ $=15 \mathrm{~nm}$ and $H 1=H 2=2 \mathrm{~nm}$. Panel (a) shows the simulation for an unstrained system, calculated with the multiband 


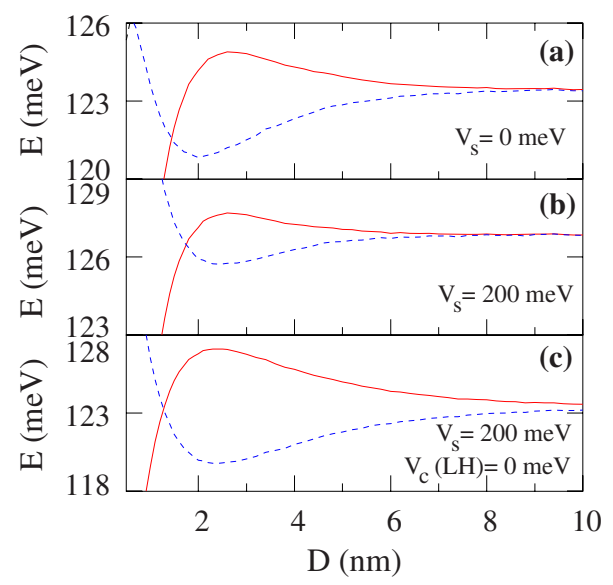

FIG. 7. (Color online) Energy levels of the two lowest hole states as a function of the interdot distance for different values of the HH-LH energy splitting due to biaxial strain, $V_{s}$. In panel (c), the interdot barrier height for LH components has been lowered to $V_{c}=0$, as expected for strained CQDs. Solid and dashed lines represent $\nu=\uparrow$ and $\nu=\downarrow$ states, respectively.

Luttinger-Kohn Hamiltonian as in the previous sections. Panel (b) shows the simulation for the same CQD but adding a strain-induced energy offset $V_{s}$ to the $\hat{P}_{-}$operators of $\hat{\mathcal{H}}_{\mathrm{LK}}$, which splits the LH components. Clearly, the qualitative behavior is not affected by the splitting since for realistic values of the strain $\left(V_{s}=200 \mathrm{meV}\right)$ only a moderate quenching of the SO signature is observed (the maximum splitting between $\nu=\downarrow$ and $\nu=\uparrow$ levels decreases from $\sim 4 \mathrm{meV}$ for $V_{s}$ $=0$ to $\sim 2 \mathrm{meV}$ for $V_{s}=200 \mathrm{meV}$ ). This is in spite of the fact that the ground state has about $97 \% \mathrm{HH}$ component.

In InAs/GaAs CQDs, the strain fields also lead to shallow interdot potential barriers for LHs. ${ }^{34,39}$ In the strong strain limit, the LH confinement in the vertical direction may resemble a wide single quantum well instead of a double well. ${ }^{24}$ To ascertain the influence of this effect on the hole tunneling, in Fig. 7(c) we plot the energy levels of a CQD with $V_{s}=200 \mathrm{meV}$ and a single-well confinement potential for $\mathrm{LH}$ components (i.e., neglecting the interdot barrier of Fig. 1 potential). By comparison with panel (b), where the LH had double well confinement, it is apparent that lowering the interdot barrier enhances the influence of SO coupling, and hence the negative effective tunneling elements. This is because the energy of the LH components is reduced, which increases their relative weight in the spinor. Moreover, the energy spacing between antisymmetric and symmetric LH components is reduced so that the $J_{z}=+1 / 2$ (antisymmetric) component of $\nu=\uparrow$ states becomes much larger than in unstrained states. As a result, the magnitude of $\hat{S}$ terms increases.

From the above discussions, we conclude that a correct description of hole tunneling in strained CQDs requires taking into account the strong modulation of the tunneling element induced by SO interaction.

So far we have considered symmetric CQDs. However, in most cases, vertically coupled self-assembled dots are asymmetric in size and composition, the asymmetry being an accidental consequence of the Stranski-Krastanow growth or

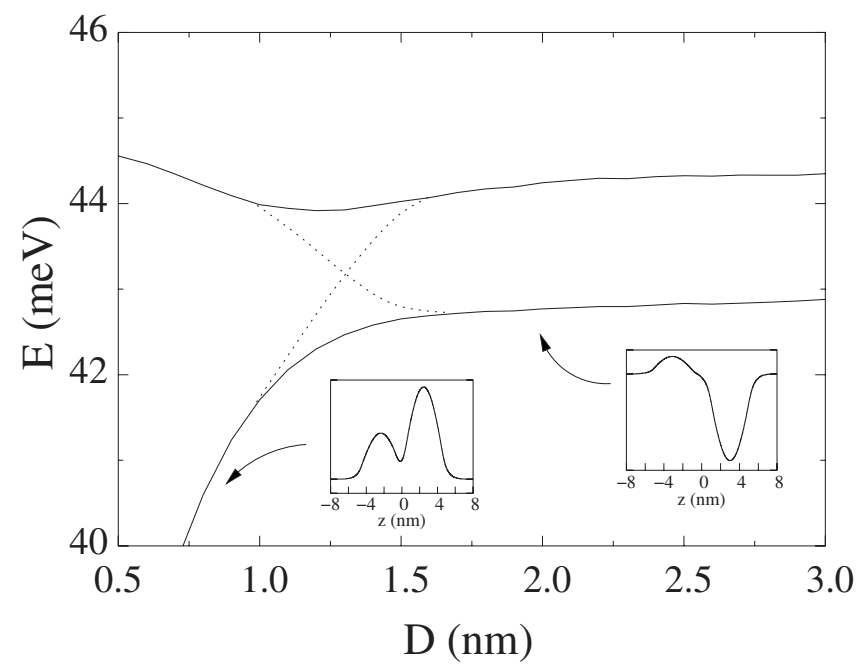

FIG. 8. Energy levels of the two lowest hole states as a function of the interdot distance in an asymmetric CQD. Dotted lines are a guide to the eye showing the expected level evolution without the anticrossing. The insets depict the wave function along $z$ for the ground-state $J_{z}=+3 / 2$ component at $D=1 \mathrm{~nm}$ and $D=2 \mathrm{~nm}$.

introduced intentionally. ${ }^{6}$ Different effects of the broken symmetry on the properties of CQDs have been studied using single-band ${ }^{17}$ and atomistic ${ }^{25}$ models. To study its influence on hole tunneling, in Fig. 8 we depict the ground and first excited states of a CQD where the coupled disks have slightly different heights, $H 1=4.1 \mathrm{~nm}$ and $H 2=4.0 \mathrm{~nm}$, and radius $R=20 \mathrm{~nm}$. In this case the chirality symmetry is broken, the spinor components having mixed symmetric and antisymmetric character. As a result, instead of a crossing between the two lowest states an anticrossing is observed. This is confirmed by the insets representing the ground-state dominant component $\left(J_{z}=+3 / 2\right)$ before and after the anticrossing. One can see that the ground state still changes from mostly bonding to mostly antibonding as $D$ increases. ${ }^{40}$ Therefore, hole tunneling in asymmetric CQDs also displays the characteristic behavior arising from SO coupling.

It is now possible to apply external electric fields upon asymmetric CQDs in order to form coherent molecular states. ${ }^{4-9,22}$ These states are achieved by bringing the levels of either electrons or holes in the upper and lower dots into resonance so that the resonant particle becomes delocalized over the entire structure. When resonant hole states are produced, multiband $k \cdot p$ and atomistic theories predict the same tunneling behavior as described here for symmetric CQDs [Fig. 5(c)], with the tunnel matrix element collapsing at a critical interdot distance $D_{c}{ }^{22}$ This is surprising because the chirality symmetry is broken and one would expect the two lowest levels to anticross as in Fig. 8. However, the resonant electric field lifts the mixing between symmetric and antisymmetric character in the spinor components, thus restoring an effective chirality for the Luttinger spinors of the resonant hole levels.

An expected experimental manifestation of this chiral-like response is that the anticrossing gap between resonant bonding and antibonding hole states, $\Delta_{h}=2|t|$, should first decrease with the interdot distance, then collapse at $D_{c}$, next 
recover, and finally vanish asymptotically. Although conclusive proof of this behavior has not been found yet, recent data suggest a deviation from the monotonic exponential decay of $\Delta_{h}$ predicted by single-band theory. ${ }^{6,41}$

\section{EXCITON STATES AND OPTICAL TRANSITIONS}

Most theoretical simulations to date have modeled excitons in CQDs as HH excitons. ${ }^{1,3-6,8,9,11,14-19}$ In this section, we show features arising from the SO-induced subband mixing of valence holes. We consider the case of symmetric InGaAs CQDs where both electron and hole states form coherent delocalized states. We calculate the exciton energy levels and their emission spectrum. The exciton Hamiltonian is given by

$$
\hat{\mathcal{H}}_{\mathrm{exc}}=\hat{\mathcal{H}}_{e}+\hat{\mathcal{H}}_{\mathrm{LK}}-\frac{e^{2}}{\epsilon^{*}\left|\mathbf{r}_{e}-\mathbf{r}_{h}\right|} .
$$

In the above expression, $\hat{\mathcal{H}}_{e}$ is the electron Hamiltonian, $\hat{\mathcal{H}}_{\text {LK }}$ is the Luttinger-Kohn Hamiltonian for the hole, Eq. (1), and the third term is the Coulomb attraction with $e$ standing for the electron charge and $\epsilon^{*}=12.9$ for the effective dielectric constant. The electron Hamiltonian in the effective-mass approximation is

$$
\hat{\mathcal{H}}_{e}=-\frac{\hbar^{2}}{2 m^{*}}\left(\hat{p}_{\perp}^{2}+\hat{p}_{z}^{2}\right)+\hat{V}^{e}(\rho, z),
$$

where $m^{*}=0.05 m_{0}$ is the electron effective mass $\left(m_{0}\right.$ is the free-electron mass) and $\hat{V}^{e}(\rho, z)$ is the electron confinement potential, which we define as $\hat{V}^{h}(\rho, z)$, but using the conduction-band offset (we take $V_{c}=530 \mathrm{meV}$ within the usual range of strained InGaAs/GaAs heterostructures. ${ }^{32}$ ) In symmetric CQDs, the electron states have a well-defined parity along the $z$ direction, either symmetric $\left(\nu_{z}=\mathrm{s}\right)$ or antisymmetric $\left(\nu_{z}=\right.$ as). Hamiltonian (17) is solved by exact diagonalization, yielding eigenfunctions of the form

$$
\left|n, m_{z}, \nu_{z}\right\rangle\left|s_{z}\right\rangle=\sum_{l} A_{l}^{n, m_{z}, \nu_{z}} f_{m_{z}, n}(\rho, \theta) \xi_{l}(z)\left|s_{z}\right\rangle,
$$

with $s_{z}$ standing for the spin $z$ projection. Here the summation runs over odd (even) values of $l$ for $\nu_{z}=\mathrm{s}\left(\nu_{z}=\right.$ as $)$ states. The two lowest electron states are the first symmetric and antisymmetric ones, $|+\rangle_{e}=\left|n=0, m_{z}=0, \nu_{z}=\mathrm{s}\right\rangle$ and $|-\rangle_{e}=\mid n$ $=0, m_{z}=0, \nu_{z}=$ as $\rangle$, respectively.

Electron and hole states are computed on a basis with up to six radial nodes $(n=0-5)$ and 30 harmonics in the vertical direction $(l=1-30)$. The exciton Hamiltonian is diagonalized on a basis composed by the Hartree products of the two lowest electron states (symmetric and antisymmetric) with $s_{z}=1 / 2$ and the two lowest hole states (chirality up and down) with $F_{z}=3 / 2:|+\rangle_{e}|\uparrow\rangle_{h},|+\rangle_{e}|\downarrow\rangle_{h},|-\rangle_{e}|\uparrow\rangle_{h}$, and $|-\rangle_{e}|\downarrow\rangle_{h}$.

It can be shown that, as in the single-band approximation, ${ }^{3,14}|+\rangle_{e}|\uparrow\rangle_{h}$ and $|-\rangle_{e}|\downarrow\rangle_{h}$ are optically active (bright) states, while $|+\rangle_{e}|\downarrow\rangle_{h}$ and $|-\rangle_{e}|\uparrow\rangle_{h}$ are optically inactive (dark). Let us examine a pair of instances. Since photons carry one quantum of angular momentum, in principle

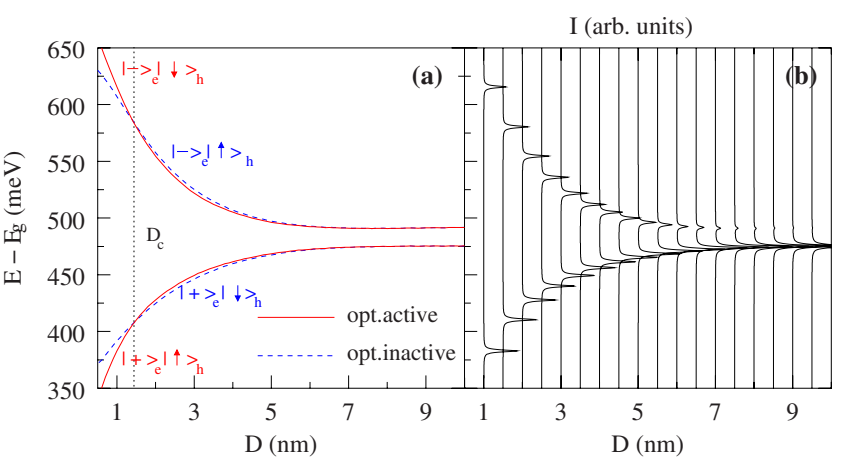

FIG. 9. (Color online) (a) Energy levels of the four lowest exciton states and (b) their corresponding optical resonances as a function of the interdot distance. $E_{g}$ stands for the gap energy. In (a), the states are labeled by their dominant single-particle component and a dot line is used to indicate the distance at which the ground-state symmetry changes.

the electron $\left|s_{z}=+1 / 2\right\rangle$ states (total angular momentum $J_{z}$ $=+1 / 2$ ) may recombine with holes through the $J_{z}=+3 / 2$ and $J_{z}=-1 / 2$ components. Additionally, one has to consider the orbital selection rules, given by the overlap between the envelope part of the electron and hole wave functions,

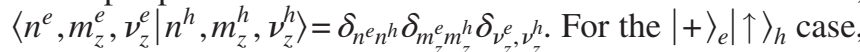
where $m_{z}^{e}=0$ and $\nu_{z}^{e}=\mathrm{s}$, the $J_{z}^{z}=-1 / 2$ component of the hole state does not fulfill the orbital selection rules, as it has $m_{z}^{h}$ $=2$ [recall Eq. (6)]. However, the $J_{z}=+3 / 2$ component fulfills the rules $\left(m_{z}^{h}=0\right.$ and $\left.\nu_{z}^{h}=\mathrm{s}\right)$, and therefore the dipole transition is possible (i.e., $|+\rangle_{e}|\uparrow\rangle_{h}$ is a bright state). On the contrary, for $|+\rangle_{e}|\downarrow\rangle_{h}$, neither the $J_{z}=+3 / 2$ nor the $J_{z}$ $=-1 / 2$ components (both with $\nu_{z}^{h}=$ as) fulfill the orbital selection rules, and the state is dark. The optically bright and dark states subspaces defined above are not mixed by Coulomb interaction.

Figure 9(a) shows the exciton energy levels as a function of the interdot distance for a CQD with $R=10 \mathrm{~nm}$ and $H 1$ $=H 2=2 \mathrm{~nm}$. The states are labeled by the dominant component of the exciton wave function. The behavior roughly resembles that estimated with single-band approximations [cf., dashed lines of Fig. 3(a) in Ref. 17]: the electron tunneling elements are much larger than the hole ones, so the pair of levels associated with $|+\rangle_{e}$ are lower in energy than those associated with $|-\rangle_{e}$. With increasing interdot distance, the tunneling elements become smaller and the two pairs of levels approach each other until they reach a constant energy splitting given by the Coulomb interaction. ${ }^{3,14}$ Nonetheless, a qualitative difference with respect to single-band results can be found within each pair of levels. Namely, the states with $|\nu=\uparrow\rangle$ and $|\nu=\downarrow\rangle$ hole component cross at a distance $D_{c}$ (dotted line in the figure). This is due to the negative hole tunneling elements described in the previous section, which alters the energy structure of excitons as well. As a matter of fact, it introduces a symmetry change in the exciton ground state from $|+\rangle_{e}|\uparrow\rangle_{h}$ to $|+\rangle_{e}|\downarrow\rangle_{h}$, which cannot be predicted without considering the hole SO coupling.

Figure 9(b) shows the emission spectrum arising from the exciton levels of panel (a). The emission intensity coming from an exciton state with energy $E$ is calculated from Fermi's golden rule as ${ }^{20}$ 


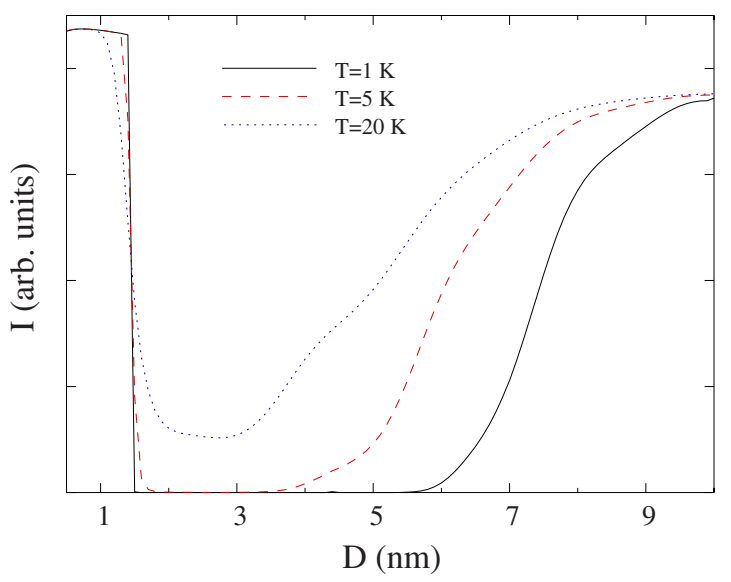

FIG. 10. (Color online) Emission intensity at thermal equilibrium as a function of the interdot distance. The CQD is the same as in Fig. 9. A strong suppression of the intensity takes place as the exciton ground state becomes dark at $D=D_{c}=1.4 \mathrm{~nm}$.

$$
I(E) \propto \sum_{i}\left|\int d \mathbf{r} \Psi_{i}^{\mathrm{exc}}(\mathbf{r}, \mathbf{r})\right|^{2} S\left(E-E_{i}\right),
$$

where $\Psi_{i}^{\mathrm{exc}}\left(\mathbf{r}_{e}, \mathbf{r}_{h}\right)$ represents the $i$ exciton state wave function with energy $E_{i}$, and $S\left(E-E_{i}\right)$ is a Lorentzian curve with band width $\Gamma=1 \mathrm{meV}$. As expected, only two resonances are observed in the spectrum, corresponding to the optically active states. Dark states remain dark in spite of the subband mixing.

In Fig. 9(b), the emission due to electron-hole recombination from both bonding and antibonding electron levels is considered. While this may be the case in CQDs subject to phonon bottleneck, ${ }^{42}$ under certain experimental conditions, nonradiative relaxation between exciton levels of the CQD may be faster than electron-hole recombination. In the latter case, low-power photoluminescence emission arises from recombination between the thermally accessible states only (mainly the exciton ground state). ${ }^{42,43}$ Therefore, the formation of a dark exciton ground state, reported in Fig. 9(a), should quench the emission intensity dramatically. To illustrate this point, we calculate the integrated emission intensity vs interdot distance assuming thermal equilibrium,

$$
I(T) \propto \frac{\sum_{i}\left|\int d \mathbf{r} \Psi_{i}^{\mathrm{exc}}(\mathbf{r}, \mathbf{r})\right|^{2} e^{-\left(E_{i}-E_{\mathrm{gs}}\right) / k_{B} T}}{\sum_{i} e^{-\left(E_{i}-E_{\mathrm{gs}}\right) / k_{B} T}} .
$$

Here $T$ is the temperature, $E_{\mathrm{gs}}$ is the exciton ground-state energy, and $k_{B}$ is the Boltzmann constant. The resulting emission intensity vs interdot distance is depicted in Fig. 10 at different temperatures. As can be seen, the intensity experiences a strong suppression as $D$ exceeds the critical distance $D_{c}=1.4 \mathrm{~nm}$ where the exciton ground state becomes dark due to the negative hole tunneling elements. For larger interdot distance, the intensity is partially recovered because the bright and dark exciton states associated with $|+\rangle_{e}$ become quasidegenerate [see Fig. 9(a)] so that the thermal oc- cupation of the optically active state increases. From this behavior, it follows that the luminescence of CQD devices is optimized for interdot distances $D<D_{c}$. It is also worth noting the opposite temperature dependence of the emission intensity before and after the change in ground-state symmetry: when $D<D_{c}$, the first excited state is dark, so increasing the temperature reduces the overall emission; conversely, when $D>D_{c}$, the first excited state is bright, and increasing the temperature enhances the emission. This could serve as an experimental signature of the exciton energy structure in CQDs.

The suppression of the emission intensity reported in this section should be expected for symmetric CQDs, where both electrons and holes are delocalized over the entire structure. With increasing structural asymmetry, the particles tend to localize in a single dot and the emission suppression becomes weaker. This will also be the case in asymmetric CQDs subject to resonant electric fields where only one of the particles is significantly delocalized.

\section{CONCLUSIONS}

We have shown that the inclusion of SO coupling and HH-LH mixing leads to effects in the tunneling of holes in CQDs. The simplest correct description of hole states is given by Luttinger spinors, obtained from a four-band $k \cdot p$ Hamiltonian. Within this model, parity symmetry in the vertical direction is no longer a good quantum number, as Luttinger spinors are composed by a mixture of components with different parities. Alternatively, we have proposed chirality as a good quantum number.

The spinors in CQDs have a strongly dominant $\mathrm{HH}$ component, which determines the tunneling matrix element for small interdot distances. However, the minor components of the spinor introduce a correction to the tunneling element, which becomes important as the interdot distance increases. This is due to the fact that in Luttinger spinors with a given chirality the parity of the most important LH component is opposite to that of the dominant $\mathrm{HH}$ component. For large interdot barriers, tunneling of LHs is stronger than that of $\mathrm{HHs}$, and therefore the effect of the LH component is greatly enhanced. As a consequence, an effective tunneling element is obtained, which may be strongly reduced as compared to the simple $\mathrm{HH}$ expectation. For large interdot distance, the effective tunneling element becomes negative and the hole ground state changes chirality symmetry (from almost symmetric to almost antisymmetric). This finding shows that the bonding to antibonding hole ground-state reversal observed in recent experiments with asymmetric CQDs under resonant electric fields ${ }^{22}$ can be explained from the Luttinger spinor nature of valence holes without the need of invoking structural asymmetries or atomistic effects.

SO effects on the exciton energy structure and optical transitions of CQDs have been also analyzed. It has been shown that they lead to dark exciton ground states, which may be reflected as a strong suppression of the CQD emission intensity at certain interdot distances. 


\section{ACKNOWLEDGMENTS}

We thank D. Gammon, M. Doty, G. Bryant, and M. Bayer for very useful suggestions, and $\mathrm{S}$. Benatti for technical assistance. We acknowledge support from FIRB-MIUR ItalyCanada Grant No. RBIN06JB4C, Italian MIUR-PRIN 2006 Grant No. 2006022932, QuantumWorks and Canadian Institute for Advanced Research (P.H., M.K.), Marie Curie Project No. MEIF-CT-2006-023797 (J.I.C.), and Cineca Calcolo Parallelo 2007.

\section{APPENDIX A: SIMPLE MODEL OF THE SO CORRECTION TO THE TUNNELING ELEMENT}

In this appendix we develop a simplified model that provides a basic insight into the effect of the SO interaction on the tunneling matrix elements of CQDs. To this end, we approximate the Luttinger-Kohn Hamiltonian for a CQD molecule as

$$
\hat{\mathcal{H}}=\hat{\mathcal{H}}_{\mathrm{QD} 1}+\hat{\mathcal{H}}_{\mathrm{QD} 2}+\hat{\mathcal{H}}_{t}+\hat{\mathcal{H}}_{\mathrm{so}}
$$

where $\hat{\mathcal{H}}_{\mathrm{QD} 1}$ and $\hat{\mathcal{H}}_{\mathrm{QD} 2}$ are single-band Hamiltonians of the bottom and top QDs. $\hat{\mathcal{H}}_{t}$ adds the tunnel-coupling term and $\hat{\mathcal{H}}_{\text {so }}$ is the relevant SO interaction term mixing $\mathrm{HH}$ and $\mathrm{LH}$ levels. We solve the above Hamiltonian step by step. First, we solve $\hat{\mathcal{H}}_{\mathrm{QD} 1}$ and $\hat{\mathcal{H}}_{\mathrm{QD} 2}$ in the basis of the $\mathrm{HH}$ and $\mathrm{LH}$ levels. The atomic (localized) hole states are obtained;

$$
\hat{\mathcal{H}}_{\mathrm{QD} 1}+\hat{\mathcal{H}}_{\mathrm{QD} 2}=\left(\begin{array}{cccc}
E_{1}^{\mathrm{HH}} & 0 & 0 & 0 \\
0 & E_{2}^{\mathrm{HH}} & 0 & 0 \\
0 & 0 & E_{1}^{\mathrm{LH}} & 0 \\
0 & 0 & 0 & E_{2}^{\mathrm{LH}}
\end{array}\right) .
$$

Here $E_{1}^{\mathrm{HH}}$ and $E_{2}^{\mathrm{HH}}\left(E_{1}^{\mathrm{LH}}\right.$ and $\left.E_{2}^{\mathrm{LH}}\right)$ are the energies of the lowest $\mathrm{HH}(\mathrm{LH})$ in QD1 and QD2 respectively. Assuming that the dots are identical $\left(E_{1}=E_{2}\right)$ and adding the tunnelcoupling term, we obtain:

$$
\hat{\mathcal{H}}_{\mathrm{QD} 1}+\hat{\mathcal{H}}_{\mathrm{QD} 2}+\hat{\mathcal{H}}_{t}=\left(\begin{array}{cccc}
E_{1}^{\mathrm{HH}} & -t_{\mathrm{HH}} & 0 & 0 \\
-t_{\mathrm{HH}} & E_{1}^{\mathrm{HH}} & 0 & 0 \\
0 & 0 & E_{1}^{\mathrm{LH}} & -t_{\mathrm{LH}} \\
0 & 0 & -t_{\mathrm{LH}} & E_{1}^{\mathrm{LH}}
\end{array}\right)=\left(\begin{array}{cccc}
E_{1}^{\mathrm{HH}}-t_{\mathrm{HH}} & 0 & 0 & 0 \\
0 & E_{1}^{\mathrm{HH}}+t_{\mathrm{HH}} & 0 & 0 \\
0 & 0 & E_{1}^{\mathrm{LH}}-t_{\mathrm{LH}} & 0 \\
0 & 0 & 0 & E_{1}^{\mathrm{LH}}+t_{\mathrm{LH}} \cdot
\end{array}\right) \text {, }
$$

where $t_{\mathrm{HH}}$ and $t_{\mathrm{LH}}$ stand for the $\mathrm{HH}$ and LH tunneling matrix elements, respectively. The above levels correspond to the $\mathrm{HH}$ and $\mathrm{LH}$ bonding and antibonding states,

$$
\begin{array}{ll}
E_{b}^{\mathrm{HH}}=E_{1}^{\mathrm{HH}}-t_{\mathrm{HH}}, & E_{a}^{\mathrm{HH}}=E_{1}^{\mathrm{HH}}+t_{\mathrm{HH}}, \\
E_{b}^{\mathrm{LH}}=E_{1}^{\mathrm{LH}}-t_{\mathrm{LH}}, & E_{a}^{\mathrm{LH}}=E_{1}^{\mathrm{LH}}+t_{\mathrm{LH}} .
\end{array}
$$

Next we include the SO interaction. We know from Sec. III A that the relevant $\mathrm{SO}$ term, $\hat{S}$, mixes $\mathrm{HH}$ and $\mathrm{LH}$ states with opposite parity to form spinors with a given chirality. Thus, we define the SO coupling terms $S_{\uparrow}=\langle b, \mathrm{HH}|\hat{S}| a, \mathrm{LH}\rangle$ and $S_{\downarrow}=\langle a, \mathrm{HH}|\hat{S}| b, \mathrm{LH}\rangle$. The complete Hamiltonian reads now as

$$
\hat{\mathcal{H}}=\left(\begin{array}{cccc}
E_{b}^{\mathrm{HH}} & 0 & 0 & S_{\uparrow} \\
0 & E_{a}^{\mathrm{HH}} & S_{\downarrow} & 0 \\
0 & S_{\downarrow} & E_{b}^{\mathrm{LH}} & 0 \\
S_{\uparrow} & 0 & 0 & E_{a}^{\mathrm{LH}} \cdot
\end{array}\right) .
$$

We then obtain two states mixed by $S_{\uparrow}$ (chirality $\nu=\uparrow$ spinors) and two mixed by $S_{\downarrow}$ (chirality $\nu=\downarrow$ spinors),

$$
E_{\nu=\uparrow}^{+}=\frac{E_{b}^{\mathrm{HH}}+E_{a}^{\mathrm{LH}}}{2}+\sqrt{S_{\uparrow}^{2}+\left(\frac{1}{2}\left(E_{b}^{\mathrm{HH}}-E_{a}^{\mathrm{LH}}\right)\right)^{2}},
$$

$$
\begin{aligned}
& E_{\nu=\downarrow}^{+}=\frac{E_{a}^{\mathrm{HH}}+E_{b}^{\mathrm{LH}}}{2}+\sqrt{S_{\downarrow}^{2}+\left(\frac{1}{2}\left(E_{a}^{\mathrm{HH}}-E_{b}^{\mathrm{LH}}\right)\right)^{2}}, \\
& E_{\nu=\downarrow}^{-}=\frac{E_{a}^{\mathrm{HH}}+E_{b}^{\mathrm{LH}}}{2}-\sqrt{S_{\downarrow}^{2}+\left(\frac{1}{2}\left(E_{a}^{\mathrm{HH}}-E_{b}^{\mathrm{LH}}\right)\right)^{2}}, \\
& E_{\nu=\uparrow}^{-}=\frac{E_{b}^{\mathrm{HH}}+E_{a}^{\mathrm{LH}}}{2}-\sqrt{S_{\uparrow}^{2}+\left(\frac{1}{2}\left(E_{b}^{\mathrm{HH}}-E_{a}^{\mathrm{LH}}\right)\right)^{2}} .
\end{aligned}
$$

Since the SO term is usually a perturbation as compared to the energy splitting between $\mathrm{HH}$ and LH states, the square root in the previous expressions can be Taylor-expanded to the first order. This yields

$$
\begin{aligned}
& E_{\nu=\uparrow}^{+}=E_{b}^{\mathrm{HH}}+\frac{S_{\uparrow}^{2}}{E_{b}^{\mathrm{HH}}-E_{a}^{\mathrm{LH}}}=E_{1}^{\mathrm{HH}}-t_{\mathrm{HH}}-t_{\mathrm{so}}^{\uparrow}, \\
& E_{\nu=\downarrow}^{+}=E_{a}^{\mathrm{HH}}+\frac{S_{\downarrow}^{2}}{E_{a}^{\mathrm{HH}}-E_{b}^{\mathrm{LH}}}=E_{1}^{\mathrm{HH}}+t_{\mathrm{HH}}-t_{\mathrm{so}}^{\downarrow},
\end{aligned}
$$

$$
E_{\nu=\downarrow}^{-}=E_{b}^{\mathrm{LH}}-\frac{S_{\downarrow}^{2}}{E_{a}^{\mathrm{HH}}-E_{b}^{\mathrm{LH}}}=E_{1}^{\mathrm{LH}}-t_{\mathrm{LH}}+t_{\mathrm{so}}^{\downarrow},
$$




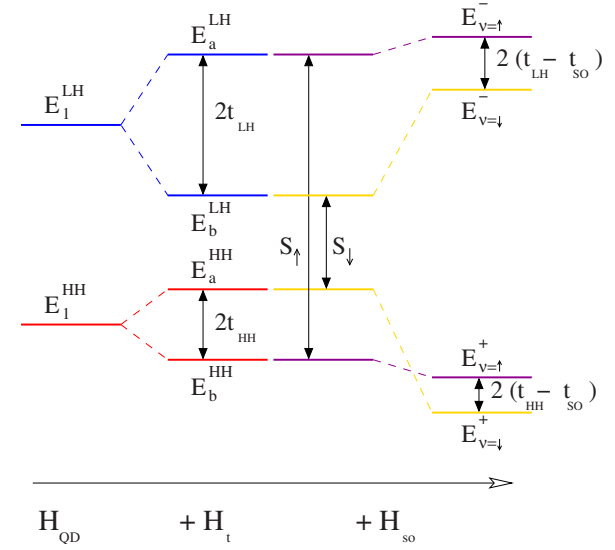

FIG. 11. (Color online) Schematic diagram of the influence exerted by each term of Hamiltonian (A1) on the energy levels of a CQD. The HH and LH levels of the QDs (left) hybridize into molecular states split by $t_{\mathrm{HH}}$ and $t_{\mathrm{LH}}$ (midleft). Spin-orbit interaction couples states with the same chirality (midright) further modifying the energy splittings (right). It is assumed that $E_{1}^{\mathrm{LH}} \gg E_{1}^{\mathrm{HH}}$ and $t_{\mathrm{LH}}$ $>t_{\mathrm{so}}>t_{\mathrm{HH}}$

$$
E_{\nu=\uparrow}^{-}=E_{a}^{\mathrm{LH}}-\frac{S_{\uparrow}^{2}}{E_{b}^{\mathrm{HH}}-E_{a}^{\mathrm{LH}}}=E_{1}^{\mathrm{LH}}+t_{\mathrm{LH}}+t_{\mathrm{so}}^{\uparrow},
$$

where we have defined the SO-induced tunneling elements $t_{\mathrm{so}}^{\uparrow}=\frac{S_{\uparrow}^{2}}{E_{a}^{\mathrm{LH}}-E_{b}^{\mathrm{HH}}}$ and $t_{\mathrm{so}}^{\downarrow}=\frac{S_{\downarrow}^{2}}{E_{b}^{\mathrm{LH}}-E_{a}^{\mathrm{HH}}}$. Equations (A5)-(A8) clearly relate the chiral spinors to molecular $\mathrm{HH}$ and $\mathrm{LH}$ states perturbed by the SO interaction. The energy splitting between the two first (mostly HH) and two last (mostly LH) states is

$$
\begin{aligned}
& E_{\nu=\downarrow}^{+}-E_{\nu=\uparrow}^{+}=2\left(t_{\mathrm{HH}}-t_{\mathrm{so}}\right), \\
& E_{\nu=\uparrow}^{-}-E_{\nu=\downarrow}^{-}=2\left(t_{\mathrm{LH}}-t_{\mathrm{so}}\right),
\end{aligned}
$$

with $t_{\mathrm{so}}=\left(t_{\mathrm{so}}^{\downarrow}-t_{\mathrm{so}}^{\uparrow}\right) / 2$. This shows that the SO perturbation acts as a correction to the HH tunneling element. Since $t_{\text {so }}^{\downarrow}$ $>t_{\text {so }}^{\uparrow}$ (because the mixed HH and LH levels are closer in energy for $\nu=\downarrow$ ), the correction tends to reduce the energy splitting. Eventually, when $t_{\mathrm{so}}$ exceeds $t_{\mathrm{HH}}$, the ground state switches from $E_{\nu=\uparrow}^{+}$(mostly a bonding $\mathrm{HH}$ state) to $E_{\nu=\downarrow}^{+}$ (mostly an antibonding $\mathrm{HH}$ state).

The results of this section are summarized diagrammatically in Fig. 11.

\section{APPENDIX B: MATRIX ELEMENTS}

The matrix elements of the operators involved in $\hat{\mathcal{H}}_{L K}$, Eqs. (2a)-(2d), can be easily obtained from the matrix elements of their momentum operators projected on the basis states $\left|n, m_{z}, l\right\rangle=f_{m_{z}, n}(\rho, \theta) \xi_{l}(z)$. For $\hat{P}_{+}$and $\hat{P}_{-}$, the constituent elements are

$$
\left\langle l_{1} m_{1} n_{1}\left|\hat{p}_{z}^{2}\right| n_{2} m_{2} l_{2}\right\rangle=\frac{\pi^{2} l^{2}}{W^{2}} \delta_{n_{1}, n_{2}} \delta_{m_{1}, m_{2}} \delta_{l_{1}, l_{2}},
$$

and

$$
\left\langle l_{1} m_{1} n_{1}\left|\hat{p}_{\perp}^{2}\right| n_{2} m_{2} l_{2}\right\rangle=\left(\frac{\alpha_{n}^{m}}{R}\right)^{2} \delta_{n_{1}, n_{2}} \delta_{m_{1}, m_{2}} \delta_{l_{1}, l_{2}},
$$

where $R$ is the radius of the disks and $W$ is the size of the computational box along $z$. For $\hat{S}$, the relevant element is

$$
\begin{aligned}
\left\langle l_{1} m_{1} n_{1}\left|\hat{p}_{-} \hat{p}_{z}\right| n_{2} m_{2} l_{2}\right\rangle= & \frac{2 \delta_{m_{1}, m_{2}-1}}{R\left|J_{m_{1}+1}\left(\alpha_{n_{1}}^{m_{1}}\right) J_{m_{2}+1}\left(\alpha_{n_{2}}^{m_{2}}\right)\right|} \\
& \times\left[-2 m_{2} \int_{0}^{1} J_{m_{1}}\left(\alpha_{n_{1}}^{m_{1}} s\right) J_{m_{2}}\left(\alpha_{n_{2}}^{m_{2}} s\right) d s\right. \\
& \left.+\alpha_{n_{2}}^{m_{2}} \int_{0}^{1} s J_{m_{1}}\left(\alpha_{n_{1}}^{m_{1}} s\right) J_{m_{2}+1}\left(\alpha_{n_{2}}^{m_{2}} s\right) d s\right] \\
& \times\left\langle l_{1}\left|\nabla_{z}\right| l_{2}\right\rangle .
\end{aligned}
$$

In the above expression, $\left\langle l_{1}\left|\nabla_{z}\right| l_{2}\right\rangle=0$ if both $l_{1}$ and $l_{2}$ are even or odd due to the odd symmetry of the integrand. Otherwise

$$
\begin{aligned}
\left\langle l_{1}\left|\nabla_{z}\right| l_{2}\right\rangle= & \frac{2 l_{2}}{W} \times\left[\frac{(-1)^{l_{2}}}{l_{1}+l_{2}} \sin \left(\frac{\left(l_{1}+l_{2}\right) \pi}{2}\right)\right. \\
& \left.+\frac{1}{l_{1}-l_{2}} \sin \left(\frac{\left(l_{1}-l_{2}\right) \pi}{2}\right)\right] .
\end{aligned}
$$

Finally, for $\hat{R}$, the required element is

$$
\begin{aligned}
\left\langle l_{1} m_{1} n_{1}\left|\hat{p}_{-}^{2}\right| n_{2} m_{2} l_{2}\right\rangle & \\
= & \frac{2 \delta_{l_{1}, l_{2}} \delta_{m_{1}, m_{2}-2}}{R^{2}\left|J_{m_{1}+1}\left(\alpha_{n_{1}}^{m_{1}}\right) J_{m_{2}+1}\left(\alpha_{n_{2}}^{m_{2}}\right)\right|} \\
& \times\left\{\left(\alpha_{n_{2}}^{m_{2}}\right)^{2} \int_{0}^{1} s J_{m_{1}}\left(\alpha_{n_{1}}^{m_{1}} s\right) J_{m_{2}}\left(\alpha_{n_{2}}^{m_{2}} s\right) d s\right. \\
& -4 m_{2}\left(m_{2}-1\right) \int_{0}^{1} \frac{1}{s} J_{m_{1}}\left(\alpha_{n_{1}}^{m_{1}} s\right) J_{m_{2}}\left(\alpha_{n_{2}}^{m_{2}} s\right) d s \\
& \left.+2 \alpha_{n_{2}}^{m_{2}}\left(m_{2}-1\right) \int_{0}^{1} J_{m_{1}}\left(\alpha_{n_{1}}^{m_{1}} s\right) J_{m_{2}+1}\left(\alpha_{n_{2}}^{m_{2}} s\right) d s\right\} .
\end{aligned}
$$

For the analytical model developed in Sec. III A, the vertical parts of the matrix elements are given by

$\left\langle\xi_{\mathrm{HH}}^{\mathrm{s}(\mathrm{as})}\left|\hat{V}^{h}\right| \xi_{\mathrm{HH}}^{\mathrm{s}(\mathrm{as})}\right\rangle=2\left(N^{\mathrm{s}(\mathrm{as})}\right)^{2}\left(\left\langle\xi_{\mathrm{HH}}^{\mathrm{QD} 1}\left|\hat{V}^{h}\right| \xi_{\mathrm{HH}}^{\mathrm{QD} 1}\right\rangle \pm\left\langle\xi_{\mathrm{HH}}^{\mathrm{QD} 1}\left|\hat{V}^{h}\right| \xi_{\mathrm{HH}}^{\mathrm{QD} 2}\right\rangle\right)$,

$$
\begin{aligned}
\left\langle\xi_{\mathrm{HH}}^{\mathrm{s}(\mathrm{as})}\left|\hat{p}_{z}^{2}\right| \xi_{\mathrm{HH}}^{\mathrm{s}(\mathrm{as})}\right\rangle= & {\left[N^{\mathrm{s}(\mathrm{as})}\right]^{2} \alpha_{\mathrm{HH}} } \\
& \times\left\{1 \pm\left\langle\xi_{\mathrm{HH}}^{\mathrm{QD} 1} \mid \xi_{\mathrm{HH}}^{\mathrm{QD} 2}\right\rangle\left[2-\left(1+2 \alpha_{\mathrm{HH}} D_{1 / 2}^{2}\right)\right]\right\},
\end{aligned}
$$

$$
\begin{aligned}
\left\langle\xi_{\mathrm{HH}}^{\mathrm{s}(\mathrm{as})}\left|\nabla_{z}\right| \xi_{\mathrm{LH}}^{\mathrm{as}(\mathrm{s})}\right\rangle= & -2 N^{\mathrm{s}} N^{\mathrm{as}} \alpha_{\mathrm{LH}} \\
& \times\left[\left\langle\xi_{\mathrm{HH}}^{\mathrm{QD} 1}|z| \xi_{\mathrm{LH}}^{\mathrm{QD} 1}\right\rangle+D_{1 / 2}\left\langle\xi_{\mathrm{HH}}^{\mathrm{QD} 1} \mid \xi_{\mathrm{LH}}^{\mathrm{QD} 1}\right\rangle\right. \\
& \left. \pm\left(\left\langle\xi_{\mathrm{HH}}^{\mathrm{QD} 2}|z| \xi_{\mathrm{LH}}^{\mathrm{QD} 1}\right\rangle+D_{1 / 2}\left\langle\xi_{\mathrm{HH}}^{\mathrm{QD} 2} \mid \xi_{\mathrm{LH}}^{\mathrm{QD} 1}\right\rangle\right)\right],
\end{aligned}
$$


where the upper and lower sign in \pm corresponds to the $\nu=\uparrow$ and $\nu=\downarrow$ state, respectively. These expressions show that states with different chirality will have different matrix elements not only for the diagonal operators of the Hamiltonian $\left[\hat{V}^{h}(z)\right.$ and $\left.\hat{p}_{z}^{2}\right]$ but also for the off-diagonal spin-orbit ones $\left(\hat{p}_{z}\right)$.

*pawel.hawrylak@nrc.ca

${ }^{1}$ G. W. Bryant, Phys. Rev. B 47, 1683 (1993).

${ }^{2}$ J. J. Palacios and P. Hawrylak, Phys. Rev. B 51, 1769 (1995).

${ }^{3}$ M. Bayer, P. Hawrylak, K. Hinzer, S. Fafard, M. Korkusinski, Z. R. Wasilewski, O. Stern, and A. Forchel, Science 291, 451 (2001).

${ }^{4}$ G. Ortner, M. Bayer, Y. Lyanda-Geller, T. L. Reinecke, A. Kress, J. P. Reithmaier, and A. Forchel, Phys. Rev. Lett. 94, 157401 (2005).

${ }^{5}$ E. A. Stinaff, M. Schneibner, A. S. Bracker, I. V. Ponomarev, V. L. Korenev, M. E. Ware, M. F. Doty, T. L. Reinecke, and D. Gammon, Science 311, 636 (2006).

${ }^{6}$ A. S. Bracker, M. Schneiber, M. F. Doty, E. A. Stinaff, I. V. Ponomarev, J. C. Kim, L. J. Whitman, T. L. Reinecke, and D. Gammon, Appl. Phys. Lett. 89, 233110 (2006).

${ }^{7}$ M. F. Doty, M. Scheibner, I. V. Ponomarev, E. A. Stinaff, A. S. Bracker, V. L. Korenev, T. L. Reinecke, and D. Gammon, Phys. Rev. Lett. 97, 197202 (2006)

${ }^{8}$ H. J. Krenner, M. Sabathil, E. C. Clark, A. Kress, D. Schuh, M. Bichler, G. Abstreiter, and J. J. Finley, Phys. Rev. Lett. 94, 057402 (2005).

${ }^{9}$ H. J. Krenner, E. C. Clark, T. Nakaoka, M. Bichler, C. Scheurer, G. Abstreiter, and J. J. Finley, Phys. Rev. Lett. 97, 076403 (2006).

${ }^{10}$ C. P. Garcia, S. Kalliakos, V. Pellegrini, A. Pinczuk, B. S. Dennis, L. N. Pfeiffer, and K. W. West, Appl. Phys. Lett. 88, 113105 (2006).

${ }^{11}$ For a review, see e.g. H. J. Krenner, S. Stufler, M. Sabathil, E. C. Clark, P. Ester, M. Bichler, G. Abstreiter, J. J. Finley, and A. Zrenner, New J. Phys. 7, 184 (2005).

${ }^{12}$ F. Troiani, E. Molinari, and U. Hohenester, Phys. Rev. Lett. 90, 206802 (2003).

${ }^{13}$ E. Biolatti, I. D'Amico, P. Zanardi, and F. Rossi, Phys. Rev. B 65, 075306 (2002).

${ }^{14}$ M. Korkusinski, P. Hawrylak, M. Bayer, G. Ortner, A. Forchel, S. Fafard, and Z. Wasilewski, Physica E (Amsterdam) 13, 610 (2002).

${ }^{15}$ Q. R. Dong, S. S. Li, Z. C. Niu, and S. L. Feng, Physica E (Amsterdam) 33, 230 (2006).

${ }^{16}$ W. Chu and J. L. Zhu, Appl. Phys. Lett. 89, 053122 (2006).

${ }^{17}$ J. L. Zhu, W. Chu, Z. Dai, and D. Xu, Phys. Rev. B 72, 165346 (2005).

${ }^{18}$ Y. B. Lyanda-Geller, T. L. Reinecke, and M. Bayer, Phys. Rev. B 69, 161308(R) (2004).

${ }^{19}$ B. Szafran, S. Bednarek, and J. Adamowski, Phys. Rev. B 64, 125301 (2001).

${ }^{20}$ L. Jacak, P. Hawrylak, and A. Wojs, Quantum Dots (SpringerVerlag, Berlin, 1998).

${ }^{21}$ J. I. Climente, M. Korkusinski, G. Goldoni, and P. Hawrylak, Physica E (Amsterdam) 40, 1862 (2008).

${ }^{22}$ M. F. Doty, J. I. Climente, M. Korkusinski, M. Scheibner, A. S. Bracker, P. Hawrylak, and D. Gammon, arXiv:0804.3097 (unpublished).
${ }^{23}$ W. Jaskólski, M. Zieliński, and G. W. Bryant, Acta Phys. Pol. A 106, 193 (2004).

${ }^{24}$ W. Jaskólski, M. Zieliński, G. W. Bryant, and J. Aizpurua, Phys. Rev. B 74, 195339 (2006).

${ }^{25}$ G. Bester, J. Shumway, and A. Zunger, Phys. Rev. Lett. 93, 047401 (2004); G. Bester, A. Zunger, and J. Shumway, Phys. Rev. B 71, 075325 (2005).

${ }^{26}$ M. Korkusinski, W. Sheng, P. Hawrylak, Z. Wasilewski, G. Ortner, M. Bayer, A. Babinski, and M. Potemski, Proceedings of the 27th International Conference on the Physics of Semiconductors, 2005 (unpublished), p. 685.

${ }^{27}$ J. M. Luttinger and W. Kohn, Phys. Rev. 97, 869 (1955).

${ }^{28}$ L. C. Andreani, A. Pasquarello, and F. Bassani, Phys. Rev. B 36, 5887 (1987).

${ }^{29}$ L. G. C. Rego, P. Hawrylak, J. A. Brum, and A. Wojs, Phys. Rev. B 55, 15694 (1997).

${ }^{30}$ The split-off states not only are farther in energy but their mass is not as light as that of LHs which, as we explain below, is central to originate the characteristic behavior of holes in CQDs.

${ }^{31}$ Z. R. Wasilewski, S. Fafard, and J. P. McCaffrey, J. Cryst. Growth 201-202, 1131 (1999).

${ }^{32}$ I. Vurgaftman, J. R. Meyer, and L. R. Ram-Mohan, J. Appl. Phys. 89, 5815 (2001).

${ }^{33}$ In the absence of external magnetic fields, $\left|F_{z}, \nu=\uparrow, k\right\rangle$ and $\mid$ $\left.-F_{z}, \nu=\downarrow, k\right\rangle$ are degenerate (Kramer doublets) (Ref. 29). For example, the ground state $|3 / 2, \uparrow, 1\rangle$ is degenerate with $-3 / 2, \downarrow, 1\rangle$. For simplicity of the discussion, in the main text we only discuss the $F_{z}=3 / 2$ states. The reasoning for $F_{z}=-3 / 2$ would be analogous.

${ }^{34}$ M. Korkusinski and P. Hawrylak, Phys. Rev. B 63, 195311 (2001).

${ }^{35}$ For the $F_{z}=-3 / 2$ Kramer-degenerate states the relationship is reversed: $\nu=\uparrow$ corresponds to "almost antisymmetric" and $\nu=\downarrow$ to "almost symmetric."

${ }^{36}$ For very strong tunneling, the symmetric LH may as well become the dominant component of the spinor, see Q. Zhu, F. Karlsson, A. Rudra, E. Pelucchi, and E. Kapon, Physica E (Amsterdam) 40, 1815 (2007).

${ }^{37}$ Q. Xie, A. Madhukar, P. Chen, and N. P. Kobayashi, Phys. Rev. Lett. 75, 2542 (1995).

${ }^{38}$ C. Pryor, Phys. Rev. B 57, 7190 (1998).

${ }^{39}$ C. Pryor, Phys. Rev. Lett. 80, 3579 (1998).

${ }^{40}$ By analogy to atomic physics, we define as bonding (antibonding) molecular states those having zero (one) node in the direction of molecular coupling. For identical molecules, this is equivalent to symmetric (antisymmetric) parity states.

${ }^{41}$ M. F. Doty and D. Gammo (private communication).

${ }^{42}$ G. Ortner, R. Oulton, H. Kurtze, M. Schwab, D. R. Yakovlev, M. Bayer, S. Fafard, Z. Wasilewski, and P. Hawrylak, Phys. Rev. B 72, 165353 (2005).

${ }^{43}$ I. Shtrichman, C. Metzner, B. D. Gerardot, W. V. Schoenfeld, and P. M. Petroff, Phys. Rev. B 65, 081303(R) (2002). 